\title{
PREMIUM CALCULATION IMPLICATIONS OF REINSURANCE WITHOUT ARBITRAGE
}

\author{
By GARY G. Venter \\ Workers' Compensation Reinsurance Bureau, Hoboken, New Jersey
}

\begin{abstract}
Constraints imposed on premium calculation principles are studied under one aspect of competitive market theory: the impossibility of systematic arbitrage. Principles based on second moments or utility theory are shown to lead to arbitrage possibilities, while some other principles do not.
\end{abstract}

\section{KEYWORDS}

Premium calculation; arbitrage; utility theory; capital asset pricing model.

\section{INTRODUCTION}

Insurers are in business to make a profit, and risk theory has shown that a profit margin is in fact required by prudent insurers. How to build into different insureds' premiums margins which rightly reflect relative riskiness is the topic of Premium Calculation Principles. For instance, the expected value principle adds a constant percentage load to each contract, while the standard deviation and variance principles incorporate loads proportional to the second central moment, or its square root.

In this paper it is hypothesized that at any one time there is a market premium calculation principle operant, and that market forces require its use by all insurers and reinsurers, wittingly or not. It is further assumed that a market is available for any risk priced according to this principle. One aspect of competitive market theory, namely that arbitrage profit possibilities are quickly extinguished by market competition, will then be used to place constraints on what this market premium calculation principle could be. Essentially, principles will be ruled out by showing how a portfolio of direct, assumed, and ceded policies could be assembled to create an arbitrage profit if that principle were the market principle. A class of premium principles consistent with no arbitrage will be identified.

Results using the theory of arbitrage free markets to price financial assets were given by HARRISON and KREPS (1979). MERTON (1973) showed how options can be priced through this approach. Generally the theory is carried out assuming that transaction costs will have minimal effects. This will also be assumed below, and so the results are strictly applicable only under this assumption. However, some consideration as to the possible impacts of non-negligible transactions costs are addressed. 


\section{CONSTRAINTS ON PREMIUM PRINCIPLES}

\subsection{Empirical constraint}

First, an empirical observed constraint will be employed: a premium calculation principle should produce a higher load, relative to expected losses, for an excess of loss cover than for a primary cover on the same risks. This constraint thus automatically rules out an expected value load. Further observational evidence on relative premiums for excess of loss covers by layer will be proposed below as tests of remaining principles.

\subsection{Arbitrage constraints}

The first constraint imposed by arbitrage considerations is additivity for independent risks. This is illustrated by an example. Loss experience for a group of 100 truck drivers who band together to buy insurance will be relatively more predictable than for one of the truckers alone, i.e., probable deviations from expected results will be smaller. Because of the greater uncertainty, an insurer may want to give a single trucker a proportionally larger load than the 100 together.

If this happens as a general market practice, however, a reinsurance arbitrage possibility is created. Reinsurers could assume the liability on the single truckers for lower risk premium than the insurers charged, guaranteeing those insurers a risk free profit, and then cede groups of truckers for a still lower premium that is nonetheless higher than the market would charge for such a group, thereby achieving a risk free profit themselves and an above market premium for their retrocessional markets.

In general, the possibility of such packaging of exposures shows that a market without arbitrage must charge additive premiums for independent risks. This constraint rules out the standard deviation load as a market pricing principle. The standard deviation of risk experience would be 10 times as great for the 100 truckers than for one alone, giving them one-tenth the proportional load of a single trucker. Thus $90 \%$ of the load for individual truckers in this example would be available for arbitrage profits.

The profit available from any such reinsurance arbitrage would be reduced by transaction costs. However, given the automatic facilities available in the reinsurance market, such costs are likely to be small compared to the $90 \%$ of profit available to the cedents at no risk. The market could in fact sustain a charge to the small risk equal to their share of these transaction costs, in addition to a load proportional by risk size to the large risks' load. It is doubtful, however, that this could produce a standard deviation based load.

The second constraint is additivity for non-independent risks. Again reasoning by example, consider a retired couple who own two mobile homes in the same trailer park in Oklahoma and who want to purchase homeowners insurance. When the wind comes sweeping down the plain, both homes stand a chance of being damaged. An insurer may thus feel exposed to more than twice 
the dollar variability in results insuring both than insuring just one, and may thus want more than twice the single home premium for the two.

But the market cannot charge a two trailer surcharge, because the couple could just buy separate policies. Alternatively, the insurer could cede them separately to two reinsurers. Either alternative illustrates the requirement that market premiums be additive for non-independent risks, and thus rules out the variance principle. Otherwise, de-packaging of exposures could create arbitrage profits. The de-packaging transaction cost to the original insured could be quite small, in that two policies could probably be obtained in one visit to a broker.

The additivity requirement can also be illustrated in the realm of excess reinsurance. Layering a risk reduces the variance, as the sum of the variances of the layers is less than the variance of the whole. The covariances are positive and they disappear in the layering process. If there is a price benefit to this layering, it must be passed on to the original insureds. Otherwise, if the total price is greater than the sum of the layer prices, arbitrage possibilities are created. For instance, since we are assuming that markets exist, the insureds could buy primary and excess coverage separately, and get the price benefit for minimal transaction costs.

\section{UTILITY PRINCIPLES}

The above constraints together also rule out premiums calculated as the certainty equivalent from a utility function, as will be shown next.

If $u(s)$ is the utility of the current surplus, the certainty equivalent of a portfolio of risks with uncertain losses $X$ is that $p$ which gives $u(s)=E[u(s+p-X)]$. That is, it is the constant amount which makes one with utility function $u$ indifferent between taking both the premium and the portfolio of risk or taking neither.

A popular example is exponential utility, e.g., $u(s)=1-\exp (-s / a)$. It is not difficult to show that $p=a \ln E[\exp (X / a)]$. It follows readily from this that the certainty equivalent of a portfolio of independent risks is the sum of the certainty equivalents of the risks in the portfolio. BORCH (1968) showed that additivity for independent risks holds only for the linear and exponential utility functions. Thus additivity for independent risks rules out any others.

For correlated risks $X$ and $Y$, however, $E[\exp (X / a) \exp (Y / a)]$ is not the same as $E[\exp (X / a)] E[\exp (Y / a)]$, due to covariance, and so additivity will fail. Thus additivity for non-independent risks rules out exponential utility. Linear utility is a special case of the expected value principle, and so ruled out by empirical constraints.

\section{POSSIBLE PREMIUM PRINCIPLES}

\subsection{Introduction}

Two principles that can sometimes meet the above constraints are: 1) expected value principle applied to an adjusted probability distribution and 2) a load 
proportional to the covariance of the risk with a selected "target" variable. Since both operations are additive regardless of independence, the additivity constraints are always satisfied. That higher percentage loads for higher layers can sometimes hold as well is shown below.

\subsection{Adjusted distribution principles}

Consider for example a line of business with the (shifted) Pareto severity distribution $1-(1+x / b)^{-2}$. (For the sake of argument, assume that this distribution incorporates both process and parameter risk, if that distinction is of concern.) The expected claim size is given by $b$, and the claim size limited to $x$ is $b\left(1-(1+x / b)^{-1}\right)$. The premium calculation principle to be used is to replace $b$ by $1.1 b$ in the distribution function, and then compute the expected value of a loss under the adjusted distribution. This will be done for two covers: primary coverage up to the limit $10 b$, and excess coverage above this limit.

Note that the original severity gives an expected loss of $10 b / 11$ and $b / 11$ for these layers, respectively. Under the adjusted distribution, these become $110 \mathrm{~b} / 111$ and $12.1 \mathrm{~b} / 111$, respectively, for a total of $122.1 \mathrm{~b} / 111$, which is a $10 \%$ load overall. This breaks down as a $9 \%$ load for the primary layer and a $20 \%$ load for the excess. Although in this example, the charge was the mean from the adjusted distribution, a constant times this mean could be used as well.

This is an example of a scale transformation of a distribution. In general, if $f(x)$ is a density function and $a>0, g(x)=a f(a x)$ is a scale transformation. It $(g)$ is also a distribution function, i.e., positive and integrates to unity, which can be seen by the change of variable $y=a x$. The distribution functions are related by $G(x)=F(a x)$. A scale transformation is particularly easy to implement if the distribution has a scale parameter, like $b$ in the example above. Transforming the scale of the severity distribution produces the same scale change on aggregate losses, and this is essentially the only way to do so.

Replacing the distribution by any other distribution will satisfy the additivity constraints. A scale transformation is probably the most elementary approach to finding a revised distribution. The above example shows that this can result in higher loads for excess layers, which is the empirical constraint. A more intricate transformation is the combined scale-power transformation $g(x)=a c(a x)^{c-1} f\left((a x)^{c}\right)$, i.e., $\quad G(x)=F\left((a x)^{c}\right)$. This transformation changes an exponential distribution into a Weibull, for example, or a Pareto into a Burr.

\subsection{Covariance principles}

For the covariance case, let $G$, the price of gold, be the target variable, and let the premium for a loss variable $X$ be $a E(X)+b \operatorname{Cov}(X, G)$. Because the covariance of two variables $X$ and $Y$ with a fixed auxiliary variable is additive 
whether or not $X$ and $Y$ are independent, this satisfies the additivity constraints. Does it satisfy the empirical constraint? Presumedly $G$ is highly correlated with the inflation rate, as are the excess losses, while the primary losses are probably less so. Thus the loading factor for excess could exceed that for primary under this principle.

The price of gold may not be a reasonable target variable. CAPM theory suggests using the gains on the stock market. A more general approach is given by ANG and LAI (1987), who argue from capital market and insurance market considerations that a reasonable target variable might be the difference between total market insured losses and total investment gains on all publicly traded instruments in the economy. As they show, this overcomes some of the problems insurance practitioners have had with CAPM, and it quite possibly could give a higher percentage load to excesss losses, but this would have to be verified. However, both this and CAPM theory assume either a quadratic utility function or that risk preferences can be captured with just two moments, both which are questionable.

If by some chance the target variable turned out to be the losses on a particular insurance portfolio, then the covariance pricing principle applied to that portfolio would be the variance principle. Changing the target variable for every contract so that the variance principle would always be applied would not satisfy the arbitrage constraints, however.

\subsection{Covariance principle results from an adjusted distribution}

Another method of adjusting a distribution is to multiply the density $f(x)$ by a non-negative function $h(x)$ such that $f(x) h(x)$ integrates to unity. Quite a range of such functions could be used, for as long as the integral is finite, it can be made to be unity by applying a factor to $h$.

As an example, let $h(x)=1+b(E(Y \mid x)-E(Y))$ for some target variable $Y$, where $b$ is small enough for $h$ to be positive. Then $E(h(X))=$ $1+b(E E(Y \mid X)-E(Y))=1$, which shows that $f(x) h(x)$ is a density function, and $a E(X h(X))=a E(X)+a b(E(X Y)-E(X) E(Y))=a E(x)+a b \operatorname{Cov}(X, Y)$, which shows that the adjusted distribution principle for this $h$ is the covariance principle with target $Y$.

\subsection{Other principles}

Can every principle which satisfies additivity be expressed as an adjusted distribution principle? That is, does an additive premium principle induce a distribution function on loss random variables so that the price for any coverage can be expressed as the expected value of the losses for that coverage under that distribution?

It would seem that this could be approximated to any desired degree of accuracy, according to the following reasoning. For any $m>0$, consider the coverage $C_{m}$ which pays a small amount $d$ just in the event that losses are at least $m$. First, it would seem that any coverage could be approximated by a 
linear combination of these coverages, i.e., as $\Sigma a_{i} C_{i}$. For instance, full coverage up to some limit $M$ would be approximately $\sum_{i=1}^{M / d} C_{i d}$. This pays $d$

if losses are at least $d$, another $d$ if they are at least $2 d$, etc. This approximation gets better with smaller $d$. If there is no upper limit, the sum can go to infinity. If there are only partial payments, the coefficients $a_{i}$ would be less than 1 .

The price of the coverage $C_{m}$ will be seen to induce a probability distribution that by additivity will in turn generate the prices for all coverages. The price of $C_{m}$ given a distribution $F$ would be $d(1-F(m))$. Thus the price function induces the distribution $F(m)=1-\operatorname{Price}\left(C_{m}\right) / d$.

By additivity, the price of any layer of full coverage, i.e., $a_{i}$ 's all equal 1 , would be the sum of these terms $d(1-F(i))$, which would be the expected value under the induced distribution $F$. If the coverage is not $100 \%$ in the layer, i.e., $a_{i}$ 's $<1$, the price would have to reduce by the same percentage as the coverage, because by additivity the full coverage price would have to be the sum of the prices of the reduced coverage layer and its complementary layer. Thus the price would again be computed as the expected value under the induced distribution.

The result is that the only premium calculation principles that preserve additivity are those generated by transformed distributions. This is similar to the results of HARRISON and KREPS (1979), and later HARRISON and PLISKA (1981), who showed that in an arbitrage free market, pricing of financial instruments should take place according to the expectation under a risk adjusted probability distribution. It is also closely related to the results of Delbaen and HaEzendonck (1989). They however apparently allow the random variable being priced to enter into the probability adjustment, so that a variance load can result, which contrasts to the arbitrage free considerations above.

\section{APPLICATION}

\subsection{Minimum rates on line}

An empirical reinsurance market phenomenon is minimum rate on line. The rate on line is the premium divided by the coverage limit, and most reinsurers establish a minimum they will accept for this ratio. Although there are various ad hoc explanations for this practice, it would be interesting to see to what extent it could be explained as a form of risk load. The example below shows that this can be partially accomplished by an adjusted distribution risk load.

The above shifted Pareto distribution is similar in form but less heavy tailed than severity distributions commonly used in US casualty insurance. The adjustment below can be done with more heavy distributions with similar effect. For this distribution, the expected loss in the layer $(u, v)$ is $b^{2}(v-u) /(b+u)(b+v)$. For pricing excess coverage above $1000 b$, assume use of a charge of $1.2510^{-6}$ times the expected value from a distribution with 
$1-F(x)=(1+x / b)^{-.1}$ for $x>1000 b$. For this distribution, the layer expected value is $(b / .9)\left[(1+\mathrm{v} / b)^{9}-(1+u / b)^{9}\right]$. Take the case where $b=1000$, which has severity mean $=1000$. The expected loss per first dollar claim and the corresponding charge from this rule are shown for various $\$ 1$ million excess layers:

\begin{tabular}{rcc}
\hline Retention & Layer Expected & Layer Charge \\
\hline $1,000,000$ & .499251 & .602821 \\
$11,000,000$ & .007574 & .490740 \\
$21,000,000$ & .002164 & .460965 \\
$31,000,000$ & .001008 & .443690 \\
$41,000,000$ & .000581 & .431624 \\
$51,000,000$ & .000377 & .422405 \\
\hline
\end{tabular}

Although an absolute flat charge per million of coverage is never reached, it is closely approximated by this rule. The charges are certainly dropping off much more slowly than the expected losses. This example shows that the kind of leveling of charges seen in minimum rates on line can be produced by adjusting distributions. The key is to have a low absolute value for the negative exponent in the pricing distribution function. The layer charges and the point at which leveling off occurs can be adjusted through the $b$ parameter and the constant multiplier, here $1.2510^{-6}$.

This approach to minimum rates on line will approximate such a minimum for risks of a given size, but larger or smaller risks wili have larger or smaller rates. A true minimum rate on line applicable to all risks or treaties would seem to generate arbitrage possibilities. A reinsurer could retrocede two minimum rated risks for the price of one. In a competitive market, competition would reduce the minimums for smaller treaties to gain this retrocessional opportunity.

In a quasi-monopolistic market where these savings are not passed on to the original cedent or insured, spirals of retrocession could be generated, where $A$ retrocedes to $B$ who retrocedes to $C$ who retrocedes to $A$, etc., with an arbitrage profit being taken at each step.

\section{SUMMARY}

A general advantage of changing the distribution is that it is easy to calculate charges, at least after the adjusted distribution has been established. A particular advantage of a covariance load is that in the form of CAPM it has somewhat of an economic justification. It is not clear that arbitrage theory itself could further specify the adjustments to the distribution, however. The best test is probably empirical, i.e., what sells; life actuaries have been at this enterprise for years, adjusting mortality tables in different ways depending on whether an annuity or insurance is being marketed. The time honored practice 
of fudging the table thus has stronger justification than might have been anticipated.

\section{REFERENCES}

ANG, J.S. and LAI, T. (1987) Insurance Premium Pricing and Ratemaking in Competitive Insurance and Capital Asset Markets. Journal of Risk and Insurance 54, No. 4 (December).

Borch, K. H. (1968) The Economics of Uncertainty. Princeton University Press, Princeton, N.J.

Delbaen, F. and Haezendonck, J. (1989) A Martingale Approach to Premium Calculation Principles in an Arbitrage Free Market. Insurance: Mathematics and Economics, No. 4.

HARrison, J.M. and KrePs, D.M. (1979) Martingales and Arbitrage in Multi-period Securities Markets. Journal of Economic Theory 20.

Harrison, J.M. and Pliska, S. R. (1981) Martingales and Stochastic Integrals in the Theory of Continuous Trading. Stochastic Processes and Their Applications 11.

Merton, R.C. (1973) Theory of Rational Option Pricing. Bell Journal of Economics and Mangement Science 4, (Spring).

GARY G. VENTER

Workers' Compensation Reinsurance Bureau,

Two Hudson Place, Hoboken, NJ 07030, U.S.A. 